\title{
Larger infarct size associated with dysglycemia at the time of ST-elevation myocardial infarction is related to later presentation
}

\author{
Naveed Razvi ${ }^{1,2^{*}}$, Ben Grundy ${ }^{2}$, Leong Ng ${ }^{1,2}$, Gerry P McCann ${ }^{1,2}$, lain Squire ${ }^{1,2}$ \\ From 16th Annual SCMR Scientific Sessions \\ San Francisco, CA, USA. 31 January - 3 February 2013
}

\section{Background}

Patients with dysglycemia at the time of ST-elevation myocardial infarction (STEMI) have a worse prognosis. The reasons for this are not entirely clear. Admission hyperglycemia has been associated with larger infarct size.

The aims of this study were to examine the relation of acute and chronic glycemic state to myocardial scar and salvage characteristics in patients with reperfused STelevation myocardial infarction.

\section{Methods}

56 patients treated for first ST-elevation myocardial infarction (STEMI) without a known diagnosis of Diabetes mellitus were prospectively enrolled between January and December 2010. Glycosylated hemoglobin (HbA1c) and glucose were sampled on admission to the emergency cardiac ward. Patients underwent CMR during the index admission (median day 2, IQR 2days), with assessment of area-at-risk (STIRs), Infarct size\%, late microvascular obstruction, and left ventricular function. Population characteristics are presented in Table 1.

Patients with below and above median glucose were compared. Spearman's rank correlation was used to compare non-parametric data. Correlations with a $\mathrm{p}<0.1$ were entered into a multivariate linear regression model. Independent $\mathrm{t}$-tests were used to compare groups above and below the median for glucose and HbA1c levels. There were no significant differences between patients receiving PPCI or thrombolysis, so these were grouped for analysis.

${ }^{1}$ Cardiovascular Sciences, University of Leicester, Leicester, UK

\section{Results}

When patients were dichotomised into glucose levels below $(<7.8)$ and above the median $(\geq 7.8)$, the supramedian group were significantly older (64.7 years vs. 57.1 years, $\mathrm{p}=0.018)$ and had greater infarct size $(28.33 \%$ vs. $18.46 \%, \mathrm{p}=0.007)$.

Dichotomising patients by HbA1c into levels below the median $(<5.9 \%)$ and above the median $(\geq 5.9 \%)$, the supra-median group had significantly greater glucose levels $(8.8 \mathrm{mmol} / \mathrm{l}$ vs. $7.1 \mathrm{mmol} / \mathrm{l}, \mathrm{p}=0.010)$, lower ECG resolution $(51.6 \%$ vs. $75.5 \%, \mathrm{p}=0.018)$, greater $\mathrm{MVO} \%$ ( $2.77 \%$ vs. $1.11 \%, \mathrm{p}=0.049)$, greater IMH\% ( $1.96 \%$ vs. $0.61 \%,=-0.015)$, and lower myocardial salvage index ( $43.30 \%$ vs. $65.15 \%, \mathrm{p}=0.003$ ).

On multivariate linear regression analysis however, glucose was not a predictor of IS\% $(R=0.549, R 2=0.302$, Age $t=3.441, p=0.001$, time to reperfusion $t=2.708$, $\mathrm{p}=0.009$, glucose- NS), and HbA1c was not a predictor of MSI\% ( $R=0.453, \mathrm{R} 2=0.206$, age $\mathrm{t}=-2.529, \mathrm{p}=0.015$, time to reperfusion $\mathrm{t}=-2.237, \mathrm{p}=0.030$, HbA1c-NS).

\section{Conclusions}

Admission glucose levels are associated with larger infarct size, and HbA1c levels are associated with reduced myocardial salvage. However, glycemic status is not an independent predictor of infarct size or salvage when time to reperfusion is taken in to consideration.

\section{Funding}

Acknowledgements: This work was supported by a British Heart Foundation (BHF) project grant, and the NIHR Cardiovascular Biomedical Research Unit, Leicester, UK.

\footnotetext{
Full list of author information is available at the end of the article
}

C 2013 Razvi et al; licensee BioMed Central Ltd. This is an Open Access article distributed under the terms of the Creative Commons Attribution License (http://creativecommons.org/licenses/by/2.0), which permits unrestricted use, distribution, and reproduction in any medium, provided the original work is properly cited. 
Table 1 Patient characteristics

\begin{tabular}{|c|c|}
\hline Age (years) & $60.77+/-11.75$ \\
\hline Male & $50 / 56(89 \%)$ \\
\hline Peak CK (U/I) & $1318.8+/-1282$ \\
\hline Hypertension & $20 / 56(36 \%)$ \\
\hline Smoking & $28 / 56(50 \%)$ \\
\hline Hypercholesterolemia & $15 / 56(27 \%)$ \\
\hline Family history & 17/56 (30\%) \\
\hline Diabetes Mellitus & $0 / 56(0 \%)$ \\
\hline Reperfusion therapy: PPCI thrombolysis & $45(80 \%) 11(20 \%)$ \\
\hline Time to reperfusion (minutes) all patients PPCI only thrombolysis & $194.16+/-121.12(140) 189.36+/-113.86$ (120) $213.82+/-152.04(225)$ \\
\hline Multivessel disease & $22 / 56(39 \%)$ \\
\hline LAD culprit & $25 / 56(45 \%)$ \\
\hline Glucose $(\mathrm{mmol} / \mathrm{l})$ & $7.97+/-2.54$ (median 7.7, IQR 2.10) \\
\hline $\mathrm{HbA1c}(\%)$ & $6.67+-1.07$ (median 5.9, IQR 2.10) \\
\hline IS\% by FWHM & $23.08+/-12.97$ (median 20.31, IQR 16.68) \\
\hline $\mathrm{MVO} \%$ & $2.02+/-3.67$ (median 0.49, IQR 3.01) \\
\hline AAR\% & $41.19+/-13.78$ (median 38.78, IQR 22.21) \\
\hline MSI\% & $53.71+/-26.41$ (median 58.96, IQR 35.09) \\
\hline ECG resolution\% & $62 \%+/-34 \%$ (median 67\%) \\
\hline
\end{tabular}

$\mathrm{PPCl}=$ Primary Percutaneous Coronary Intervention, LAD= Left Anterior Descending Coronary Artery, IS=Infarct size, MVO=microvascular obstruction, $\mathrm{IMH}=$ intramyocardial hemorrhage, AAR=Area at risk, MSI= Myocardial Salvage Index.

Table 2 Correlations between infarct characteristics and glucose characteristics:

\begin{tabular}{cll}
\hline & Glucose & HbA1c \\
\hline Peak CK & $R=0.152 P=0.267$ & $R=0.120 \mathrm{P}=0.393$ \\
\hline ECG resolution \% & $\mathrm{R}=-0.393 \mathrm{P}=0.003$ & $\mathrm{R}=-0.352 \mathrm{P}=0.010$ \\
\hline Time to reperfusion & $\mathrm{R}=0.185 \mathrm{P}=0.177$ & $\mathrm{R}=0.334 \mathrm{P}=0.015$ \\
\hline Age & $\mathrm{R}=0.361 \mathrm{P}=0.007$ & $\mathrm{R}=0.184 \mathrm{P}=0.188$ \\
\hline $\mathrm{IS} \%$ & $\mathrm{R}=0.296 \mathrm{P}=0.028$ & $\mathrm{R}=0.152 \mathrm{P}=0.277$ \\
\hline MVO\% & $\mathrm{R}=0.259 \mathrm{P}=0.056$ & $\mathrm{R}=0.199 \mathrm{P}=0.152$ \\
\hline MAR\% & $\mathrm{R}=0.187 \mathrm{P}=0.176$ & $\mathrm{R}=0.292 \mathrm{P}=0.036$ \\
\hline MSI\% & $\mathrm{R}=0.128 \mathrm{P}=0.358$ & $\mathrm{R}=-0.176 \mathrm{P}=0.213$ \\
\hline
\end{tabular}

$\mathrm{IS}=$ Infarct size, $\mathrm{MVO}=$ microvascular obstruction, IMH=intramyocardial hemorrhage, AAR=Area at risk, MSI= Myocardial Salvage Index.

\section{Author details}

${ }^{1}$ Cardiovascular Sciences, University of Leicester, Leicester, UK.

${ }^{2}$ Cardiovascular Biomedical Research Unit, National Institute of Health

Research (NIHR), Leicester, UK.

Published: 30 January 2013

doi:10.1186/1532-429X-15-S1-077

Cite this article as: Razvi et al.: Larger infarct size associated with

dysglycemia at the time of ST-elevation myocardial infarction is related

to later presentation. Journal of Cardiovascular Magnetic Resonance 2013

15(Suppl 1):O77.

\section{Submit your next manuscript to BioMed Central} and take full advantage of:

- Convenient online submission

- Thorough peer review

- No space constraints or color figure charges

- Immediate publication on acceptance

- Inclusion in PubMed, CAS, Scopus and Google Scholar

- Research which is freely available for redistribution

Submit your manuscript at www.biomedcentral.com/submit 